\title{
Monogamy inequality for entanglement and local contextuality
}

\author{
S. Camalet \\ Laboratoire de Physique Théorique de la Matière Condensée, UMR 7600, \\ Sorbonne Universités, UPMC Univ Paris 06, F-75005, Paris, France
}

\begin{abstract}
We derive a monogamy inequality for entanglement and local contextuality, for any finite bipartite system. It essentially results from the relations between the entropy of a local state and the entanglement of the global state, and between the purity of a state, in the sense of majorization, and its ability to violate a given state-dependent noncontextuality inequality. We build an explicit entanglement monotone that satisfies the found monogamy inequality. An important consequence of this inequality, is that there are global states too entangled to violate the local noncontextuality inequality.
\end{abstract}

PACS numbers: 03.65.Ud, 03.65.Ta, 03.67.Mn

\section{INTRODUCTION}

One of the most important property of quantum entanglement, is known as entanglement monogamy [1, 2]. Consider two systems, say A and B, in a maximally entangled state. Since this state is pure, there is no correlation between A and any third system, say C. In this extreme case, the entanglement between $\mathrm{A}$ and $\mathrm{B}$, is maximum, and that between $\mathrm{A}$ and $\mathrm{C}$, vanishes. In the general case, there is a trade-off between the two amounts of entanglement. Expressing it in quantitative terms, requires to specify a measure of entanglement [24]. Monogamy inequalities have been derived, first for three qubits, in terms of squared concurrence [1], and then, for larger systems, and using different measures of entanglement [5-10]. Related works consider nonlocality tests based on the Clauser-Horne-Shimony-Holt (CHSH) inequality [11, 12]. When this inequality is violated for $\mathrm{A}$ and $\mathrm{B}$, it is necessarily satisfied for $\mathrm{A}$ and $\mathrm{C}$, if the same measurements are performed on $\mathrm{A}$ in both tests 13, 14. In contrast, monogamy inequalities for entanglement, do not involve specific observables, since the amount of entanglement between two systems, depends only on their common quantum state.

It has been shown that the Klyachko-Can-BinicioğluShumovski (KCBS) noncontextuality inequality [15], for A only, cannot be violated toghether with the CHSH locality inequality [16], or the $\mathcal{I}_{3322}$ inequality [17], when the same measurements are carried out on $\mathrm{A}$ in both tests. One can thus wonder whether there is a monogamy relation between entanglement and local contextuality. Such a relation must involve only the states of the global system, and of the considered local system, not particular observables. The ability of a state to disobey a noncontextuality inequality with few observables, is determined by its eigenvalues [18 21]. Moreover, if a state is less pure, in the sense of majorization [22, 23], than a state that always satisfies a noncontextuality inequality, then it also cannot violate this inequality. The purity of the state of $\mathrm{A}$, and the entanglement between $\mathrm{A}$ and $\mathrm{B}$, clearly influence each other. To see it, consider the following two extreme cases. If $\mathrm{A}$ is in a pure state, $\mathrm{A}$ and $\mathrm{B}$ are uncorrelated. If $\mathrm{A}$ and $\mathrm{B}$ are maximally entangled, the reduced density operator for A is the maximally mixed state, which is majorized by any other one.

In this paper, we derive a monogamy inequality for entanglement and local contextuality, for any finite bipartite system. To do so, we exploit the above mentioned relations between purity and contextuality, and between entanglement and local purity. We first show, in Sec. III. that, for any entanglement monotone, the entanglement between $\mathrm{A}$ and $\mathrm{B}$, cannot exceed a function of the state of A, that has the essential properties of an entropy 24]. This result expresses quantitatively how the purity of a local state, and the entanglement of the global state, constrain each other. Then, in Sec. III, we define, from any given state-dependent noncontextuality inequality, involving dichotomic observables, an entropic measure, which dictates, for a specific size of A, wether its state can disobey the inequality. Finally, in Sec. IV] we build an explicit entanglement monotone, which is upper bounded by this particular entropy function. An important consequence of the found monogamy inequality, is that there are global states too entangled to violate the local noncontextuality inequality. For four-level systems and the CHSH inequality, we obtain a simple condition, in terms of a readily computable quantity, that determines such states, in Sec. D.

\section{RELATION BETWEEN ENTANGLEMENT AND LOCAL ENTROPY}

We consider a measure $E$ of the entanglement between any two finite systems. The value $E(\rho)$, where $\rho$ is the state of the global system, consisting of the local systems $\mathrm{A}$ and $\mathrm{B}$, is positive, and vanishes if $\rho$ is not entangled. Moreover, it does not increase when two operators carry out local operations, and communicate classically. Such a function $E$ is an entanglement monotone. More specifically, $E[\Lambda(\rho)] \leq E(\rho)$ for transformations $\Lambda$ composed of local operations $\rho \mapsto \sum_{k} M_{k} \rho M_{k}^{\dagger}$, and maps of the form $\rho \mapsto \sum_{k} M_{k} \rho M_{k}^{\dagger} \otimes|k\rangle\langle k|$, where $M_{k}$ acts on one local system only, $\sum_{k} M_{k}^{\dagger} M_{k}$ is equal to the corresponding 
identity operator, and $|k\rangle$ are orthonormal states of an ancilla close to the other system [2]. We reiterate that $E$ is defined for local systems of any sizes. Clearly, the last map above transforms states of a system, into states of a different system. Moreover, $M_{k}$ can be a linear operator from the Hilbert space of a system, to that of one of its subsystems, or to that of a local larger system [3].

We are interested in the constraint on the reduced density operator of a local system, set by the entanglement $E(\rho)$. To express it, we define, for states $\rho_{\mathrm{A}}$ of a $d$-level system A,

$$
S_{d}\left(\rho_{\mathrm{A}}\right) \equiv \max _{\rho \in \mathcal{C}\left(\rho_{\mathrm{A}}\right)} E(\rho),
$$

where $\mathcal{C}\left(\rho_{\mathrm{A}}\right)$ is the set of all states $\rho$ of all composite systems consisting of $\mathrm{A}$, and another system, such that the reduced density operator for $\mathrm{A}$ is $\rho_{\mathrm{A}}$. For any system $\mathrm{B}$, and any state $\rho$ of the global system AB, consisting of $\mathrm{A}$ and $\mathrm{B}$,

$$
S_{d}\left(\operatorname{tr}_{\mathrm{B}} \rho\right) \geq E(\rho),
$$

where $\operatorname{tr}_{B}$ denotes the partial trace over B. As we will see, the equality is reached when $\rho$ is pure. We show below that the functions (11) have the essential properties of the familiar entropies (von Neumann, Rényi, Tsallis, ...) [24]. Thus, the above inequality expresses how the purity of the local state $\operatorname{tr}_{\mathrm{B}} \rho$, and the entanglement of the global state $\rho$, constrain each other. We remark that this inequality is not necessarily satisfied if $S_{d}$ is replaced by an arbitrary entropy function. For distillable entanglement, entanglement cost, entanglement of formation, and relative entropy of entanglement, eq.(11) gives the von Neumann entropy [2, 25, 26]. For robustness and negativity, $S_{d}\left(\rho_{\mathrm{A}}\right)$ is simply related to the $1 / 2$-Rényi entropy [27, 28].

Proposition 1. The functions (11) satisfy

$$
S_{d}\left(\rho_{\mathrm{A}}\right)=s(\boldsymbol{p})
$$

where $\boldsymbol{p}$ is the vector made up of the nonzero eigenvalues of $\rho_{A}$, in decreasing order, and $s$ does not depend on $d$, vanishes for $\boldsymbol{p}=1$, and obeys $s(\boldsymbol{q}) \leq s(\boldsymbol{p})$ when $\boldsymbol{q}$ majorizes $\boldsymbol{p}$.

Proof. Consider any system B', and any state $\rho \in \mathcal{C}\left(\rho_{\mathrm{A}}\right)$ of the composite system AB'. Denote its eigenvalues by $\lambda_{m}$, and its eigenstates by $\left|\psi_{m}\right\rangle$. Let us introduce a third system, say B", which constitutes, together with B', system B. Provided the Hilbert space dimension of B" is large enough, $\rho$ can be written as $\rho=\operatorname{tr}_{\mathrm{B}^{\prime \prime}}|\Psi\rangle\langle\Psi|$, where $|\Psi\rangle=\sum_{m} \sqrt{\lambda_{m}}\left|\psi_{m}\right\rangle\left|\phi_{m}\right\rangle$ is a pure state of system $\mathrm{AB}$, with orthonormal states $\left|\phi_{m}\right\rangle$ of B". As $\operatorname{tr}_{\mathrm{B}^{\prime \prime}}$ is a local operation, on $\mathrm{B}, E(\rho) \leq s$ where $s=E(|\Psi\rangle\langle\Psi|)$.

Since $\operatorname{tr}_{\mathrm{B}}|\Psi\rangle\left\langle\Psi\left|=\sum_{i} p_{i}\right| i\right\rangle\langle i|$, where $p_{i}$ are the nonzero eigenvalues of $\rho_{\mathrm{A}}$, and $|i\rangle$ are the corresponding eigenstates, $|\Psi\rangle=\sum_{i} \sqrt{p_{i}}|i\rangle\left|\chi_{i}\right\rangle$, where $\left|\chi_{i}\right\rangle$ are orthonormal states of B. For any pure state $\left|\Psi^{\prime}\right\rangle$ of $\mathrm{AB}$, with Schmidt coefficients $\sqrt{p_{i}}$, there are unitary operators $U_{\mathrm{A}}$ and $U_{\mathrm{B}}$, acting on $\mathrm{A}$ and $\mathrm{B}$, respectively, such that $\left|\Psi^{\prime}\right\rangle=U_{\mathrm{A}} \otimes U_{\mathrm{B}}|\Psi\rangle$. Thus, $|\Psi\rangle\langle\Psi|$ and $\left|\Psi^{\prime}\right\rangle\left\langle\Psi^{\prime}\right|$ can be transformed into each other by local operations. Consequently, $E\left(\left|\Psi^{\prime}\right\rangle\left\langle\Psi^{\prime}\right|\right)=s$, and hence, $s$ is a function of $\boldsymbol{p}$ only. Since $\rho$ is an arbitrary state of $\mathcal{C}\left(\rho_{\mathrm{A}}\right)$, and $|\Psi\rangle\langle\Psi| \in \mathcal{C}\left(\rho_{\mathrm{A}}\right), S_{d}\left(\rho_{\mathrm{A}}\right)=s$.

If $\boldsymbol{p}=1,|\Psi\rangle$ is a product state, and so $s=0$.

Consider $|\Phi\rangle=\sum_{i} \sqrt{q_{i}}|i\rangle\left|\chi_{i}\right\rangle$ with $\boldsymbol{q}$ majorizing $\boldsymbol{p}$. We have $s(\boldsymbol{p})=E(|\Psi\rangle\langle\Psi|)$ and $s(\boldsymbol{q})=E(|\Phi\rangle\langle\Phi|)$. Since $|\Psi\rangle\langle\Psi|$ can be changed into $|\Phi\rangle\langle\Phi|$ by local operations and classical communication [29], $s(\boldsymbol{p}) \geq s(\boldsymbol{q})$.

Relation (2) means not only that

$$
S_{d}\left(U \rho_{\mathrm{A}} U^{\dagger}\right)=S_{d}\left(\rho_{\mathrm{A}}\right),
$$

where $U$ is any unitary operator of A, but also that

$$
S_{d+1}\left(\sum_{i=1}^{d} p_{i}|\tilde{\imath}\rangle\langle\tilde{\imath}|\right)=S_{d}\left(\sum_{i=1}^{d} p_{i}|i\rangle\langle i|\right),
$$

where $\{|i\rangle\}_{i=1}^{d}$ and $\{|\tilde{\imath}\rangle\}_{i=1}^{d+1}$ are orthonormal bases of the considered Hilbert spaces, and the probabilities $p_{i}$ obey $\sum_{i=1}^{d} p_{i}=1$. The classical form of equation (44) is known as the expansibility property, and is an essential requirement for an entropic measure [24, 30].

\section{ENTROPIES FROM NONCONTEXTUALITY INEQUALITIES}

Our aim is to study the influence of the entanglement between systems A and B, on contextuality tests involving only A. This local contextuality can be revealed by considering $N$ dichotomic observables $A_{k}$ of A, such that each observable is compatible with some other ones, but not with all. We restrict ourselves to the usual case of projective measurements with two outcomes. When evaluated with a noncontextual hidden variable theory, the correlations of the compatible observables, satisfy inequalities, which can be violated by quantum states. Such a noncontextuality inequality reads

$$
\sum_{n} x_{n}\left\langle\prod_{k \in \mathcal{E}_{n}} A_{k}\right\rangle \leq 1,
$$

where $\mathcal{E}_{n}$ are subsets of $\{1, \ldots, N\}$, of any possible size, and $\langle\ldots\rangle=\operatorname{tr}\left(\rho_{\mathrm{A}} \ldots\right)$ is the average with respect to the density matrix $\rho_{\mathrm{A}}$. The observables $A_{k}$ and $A_{l}$ commute with each other when $k, l \in \mathcal{E}_{n}$. The coefficients $x_{n}$ are such that the maximum value of the left-hand side of eq.(5), is 1 for noncontextual hidden-variable models, i.e., there are $a_{k}= \pm 1$, such that $\sum_{n} x_{n} \prod_{k \in \mathcal{E}} a_{k}=1$. The familiar CHSH and KCBS inequalities [11, 12, 15], for example, can be cast into the form (5). Let us define

$$
C_{d}\left(\rho_{\mathrm{A}}\right) \equiv \sup _{\mathbf{A} \in \mathcal{A}_{d}} \operatorname{tr}\left(\rho_{\mathrm{A}} \sum_{n} x_{n} \prod_{k \in \mathcal{E}_{n}} A_{k}\right),
$$


where $d$ is the Hilbert space dimension of $\mathbf{A}, \mathbf{A}$ stands for $\left(A_{1}, \ldots, A_{N}\right)$, and $\mathcal{A}_{d}$ is the set of all $\mathbf{A}$ consisting of dichotomic observables $A_{k}$, such that $\left[A_{k}, A_{l}\right]=0$ for $k, l \in \mathcal{E}_{n}$. By construction, for a state $\rho_{\mathrm{A}}$ such that $C_{d}\left(\rho_{\mathrm{A}}\right)>1$, there are observables $A_{k}$ with which inequality (5) is violated.

It has been shown that $C_{d}\left(\rho_{\mathrm{A}}\right)=c_{d}(\boldsymbol{p})$, where $\boldsymbol{p}$ is the vector made up of the eigenvalues of $\rho_{\mathrm{A}}$, in decreasing order, and $c_{d}$ satisfies $c_{d}(\boldsymbol{q}) \geq c_{d}(\boldsymbol{p})$ when $\boldsymbol{q}$ majorizes $\boldsymbol{p}$ [20]. However, the functions $C_{d}$ do not obey the expansibility condition (44), and really depend on the dimension $d$. Due to the above-mentioned property of $c_{d}, C_{d}$ reaches its maximum, $C_{d}^{\max } \equiv c_{d}(1)$, for pure states. We assume that there are dimensions $d$ for which $C_{d}^{\max }>1$. For these values of $d$, inequality (5) constitutes a proper contextuality test, since it is not always satisfied. Note that, for some state-dependent noncontextuality inequalities, $C_{d}^{\max }$ does not depend on $d$, provided it is larger than some value [31]. For CHSH inequality, for example, it is equal to $\sqrt{2}$, for $d \geq 4$. We also remark that the operators $A_{k}=a_{k} A$, where $A$ is any dichotomic observable, and $a_{k}= \pm 1$ are such that $\sum_{n} x_{n} \prod_{k \in \mathcal{E}_{n}} a_{k}=1$, obviously fulfill the above-stated commutation relations. Such a case describes a set-up that consists of $N$ measurement apparatuses corresponding to the same observable $A$. As a consequence, if all products in eq.(5), have an even number of terms, $C_{d} \geq 1$.

To study the impact of the entanglement between $\mathrm{A}$ and $\mathrm{B}$, on the local contextuality test (5), we define

$$
S_{d}^{l c}\left(\rho_{\mathrm{A}}\right) \equiv C_{d_{0}}^{\max }-\max _{\{|\tilde{\imath}\rangle\}} t C_{d_{0}}\left(\sum_{i, j=1}^{d_{1}}\left\langle\tilde{\imath}\left|\rho_{\mathrm{A}}\right| \tilde{\jmath}\right\rangle|i\rangle\langle j| / t\right),
$$

where $d_{0}$ is a specific dimension, $d_{1}=\min \left\{d, d_{0}\right\}, t=$ $\sum_{i=1}^{d_{1}}\left\langle\tilde{\imath}\left|\rho_{\mathrm{A}}\right| \tilde{\imath}\right\rangle,\{|i\rangle\}_{i=1}^{d_{0}}$ is an orthonormal basis, and the maximum is taken over the orthonormal bases $\{|\tilde{\imath}\rangle\}_{i=1}^{d}$ of A. Since $\mathrm{C}_{d_{0}}$ obeys eq.(33), the definition (6) does not depend on any particular basis. For $d=d_{0}$, it reduces to $S_{d_{0}}^{l c}\left(\rho_{\mathrm{A}}\right)=C_{d_{0}}^{\max }-C_{d_{0}}\left(\rho_{\mathrm{A}}\right)$, but, for $d \neq d_{0}, S_{d}^{l c}$ and $C_{d}$ are not simply related to each other. As $C_{d_{0}}^{\max }$ is the maximum value of $C_{d_{0}}$, the functions (6) are positive. As a consequence of the result below, they also fulfill the properties enumerated in proposition 1. Note that there are state-independent noncontextuality inequalities 32 35] for which the definition (6) gives zero for any state, and is thus of no use. In this case, no meaningful entanglement monotone $E$ can obey eq.(11) with $S_{d}^{l c}$, since the only possibility is $E=0$. In the following, we use the notation $\boldsymbol{\lambda}(M)$ for the vector made up of the eigenvalues of the Hermitian operator $M$, in decreasing order.

Proposition 2. The functions (6) satisfy

$$
S_{d}^{l c}\left(\rho_{\mathrm{A}}\right)=C_{d_{0}}^{\max }-\sup _{\boldsymbol{\mu} \in \Lambda}\left(\sum_{i=1}^{d_{0}} \mu_{i} p_{i}\right),
$$

where $\Lambda$ is the set of all vectors $\boldsymbol{\lambda}\left(\sum_{n} x_{n} \prod_{k \in \mathcal{E}_{n}} A_{k}\right)$, with $\left(A_{1}, \ldots, A_{N}\right) \in \mathcal{A}_{d_{0}}, p_{i}=\lambda_{i}\left(\rho_{\mathrm{A}}\right)$ for $i \leq d$, and $p_{i}=0$ for $i>d$.
Proof. Consider any orthonormal bases $\{|\tilde{\imath}\rangle\}_{i=1}^{d}$ and $\{|i\rangle\}_{i=1}^{d_{0}}$, and define $\Omega=\sum_{i, j=1}^{d_{1}}\left\langle\tilde{l}\left|\rho_{\mathrm{A}}\right| \tilde{\jmath}\right\rangle|i\rangle\langle j|$, where $d_{1}=$ $\min \left\{d, d_{0}\right\}$, and the state $\omega=t^{-1} \Omega$, where $t=\operatorname{tr} \Omega$. It has been shown that $C_{d_{0}}(\omega)=\sup _{\boldsymbol{\mu} \in \Lambda}[\boldsymbol{\mu} \cdot \boldsymbol{\lambda}(\omega)]$, where $\mathbf{a} \cdot \mathbf{b}=\sum_{i=1}^{d_{0}} a_{i} b_{i}$ [20]. Since $t \boldsymbol{\lambda}(\omega)=\boldsymbol{\lambda}(\Omega)$, $t C_{d_{0}}(\omega)=\sup _{\boldsymbol{\mu} \in \Lambda}[\boldsymbol{\mu} \cdot \boldsymbol{\lambda}(\Omega)]$.

We denote $\boldsymbol{\lambda}\left(\rho_{\mathrm{A}}\right)$ by $\mathbf{p}$. For $d>d_{0}$, the matrix representation of $\Omega$, in the basis $\{|i\rangle\}$, is a diagonal block of that of $\rho_{\mathrm{A}}$, in the basis $\{|\tilde{\imath}\rangle\}$. Thus, $\mathbf{p}$ weakly submajorizes $\boldsymbol{\lambda}(\Omega)$ [23], and so, for $j=1, \ldots, d_{0}, R_{j} \equiv$ $\sum_{i=1}^{j}\left[\lambda_{i}(\Omega)-p_{i}\right]$ is negative. Consequently, for any $\boldsymbol{\mu} \in$ $\Lambda, \boldsymbol{\mu} \cdot\left[\boldsymbol{\lambda}(\Omega)-\mathbf{p}^{\left[d_{0}\right]}\right]=\sum_{j=1}^{d_{0}-1}\left(\mu_{j}-\mu_{j+1}\right) R_{j}+\mu_{d_{0}} R_{d_{0}} \leq 0$, where $\mathbf{p}^{\left[d_{0}\right]}$ is made up of the $d_{0}$ largest $p_{i}$, in decreasing order. Hence, $t C_{d_{0}}(\omega) \leq \sup _{\boldsymbol{\mu} \in \Lambda}\left(\boldsymbol{\mu} \cdot \mathbf{p}^{\left[d_{0}\right]}\right)$. For $d \leq d_{0}$, this inequality becomes an equality, with $\mathbf{p}^{\left[d_{0}\right]}$ made up of the $p_{i}$, in decreasing order, followed by $d_{0}-d$ zeros, since $\boldsymbol{\lambda}(\Omega)=\mathbf{p}^{\left[d_{0}\right]}$.

For any $d$, when $\{|\tilde{\imath}\rangle\}_{i=1}^{d}$ is such that $\rho_{\mathrm{A}}=$ $\sum_{i=1}^{d} p_{i}|\tilde{\imath}\rangle\langle\tilde{\imath}|, \boldsymbol{\lambda}(\Omega)=\mathbf{p}^{\left[d_{0}\right]}$, which finishes the proof.

\section{MONOGAMY OF ENTANGLEMENT AND LOCAL CONTEXTUALITY}

The functions (6) have all the required characteristics to satisfy eq.(11) with an entanglement monotone $E$. It remains to show that there is indeed such a measure $E$. This can be achieved, by using the convex roof method [2], since, due to the convexity of $C_{d_{0}}$ [20], $S_{d}^{l c}$, given by eq.(6), is concave.

Proposition 3. Consider, for any composite system AB, and any state $\rho$ of $\mathrm{AB}$,

$$
E^{c r}(\rho) \equiv \inf _{\left\{P_{m},\left|\Psi_{m}\right\rangle\right\} \in \mathcal{D}(\rho)} \sum_{m} P_{m} S_{d}\left(\operatorname{tr}_{\mathrm{B}}\left|\Psi_{m}\right\rangle\left\langle\Psi_{m}\right|\right),
$$

where $\mathcal{D}(\rho)$ is the set of all ensembles $\left\{P_{m},\left|\Psi_{m}\right\rangle\right\}$ such that $\sum_{m} P_{m}\left|\Psi_{m}\right\rangle\left\langle\Psi_{m}\right|=\rho, d$ is the Hilbert space dimension of A, and $S_{d}$ are positive concave functions obeying eq.(2), and vanishing for pure states.

The function $E^{c r}$ is an entanglement monotone, and satisfies eq.(1) with $S_{d}$.

Proof. We first consider that $\rho$ is not entangled. Then, by definition, $\rho$ is a mixture of pure product states $\left|\Psi_{m}\right\rangle$. The corresponding states $\operatorname{tr}_{\mathrm{B}}\left|\Psi_{m}\right\rangle\left\langle\Psi_{m}\right|$ are pure, and hence $E^{c r}(\rho)=0$.

Let us now prove that interchanging $A$ and $B$ does not modify expression (7). The reduced density operators $\operatorname{tr}_{\mathrm{B}}\left|\Psi_{m}\right\rangle\left\langle\Psi_{m}\right|$ and $\operatorname{tr}_{\mathrm{A}}\left|\Psi_{m}\right\rangle\left\langle\Psi_{m}\right|$, have the same nonvanishing eigenvalues. Thus, due to eq.(21), $S_{d}\left(\operatorname{tr}_{\mathrm{B}}\left|\Psi_{m}\right\rangle\left\langle\Psi_{m}\right|\right)$ in eq.(17), can be replaced by $S_{d^{\prime}}\left(\operatorname{tr}_{\mathrm{A}}\left|\Psi_{m}\right\rangle\left\langle\Psi_{m}\right|\right)$, where $d^{\prime}$ is the Hilbert space dimension of B.

It follows from eq.(7) that $E^{c r}$ is convex [3]. For operators $B_{k}$ of system $\mathrm{B}$, such that $\sum_{k} B_{k}^{\dagger} B_{k}$ is equal to its identity operator, the concavity of $S_{d}$ leads to 
$E^{c r}(\rho) \geq \sum_{k} p_{k} E^{c r}\left(\rho_{k}\right)$, where $p_{k}=\operatorname{tr}\left(B_{k}^{\dagger} B_{k} \rho\right)$ and $\rho_{k}=B_{k} \rho B_{k}^{\dagger} / p_{k}[3]$. This inequality and the convexity of $E^{c r}$ ensure that $E^{c r}$ does not increase under local operations on B. With expression (7) rewritten as explained above, the same proof shows that this is also the case for local operations on A. Since $\rho_{k}$ and $\tilde{\rho}_{k}=\rho_{k} \otimes|k\rangle\langle k|$, where $|k\rangle$ is a pure state of an ancilla close to A, can be transformed into each other by local operations, $E^{c r}\left(\tilde{\rho}_{k}\right)=E^{c r}\left(\rho_{k}\right)$. Thus, $E^{c r}\left(\sum_{k} p_{k} \tilde{\rho}_{k}\right) \leq E^{c r}(\rho)$, which finishes the proof that $E^{c r}$ is an entanglement monotone.

Consider a given state $\rho_{\mathrm{A}}$ of $\mathrm{A}$, and any state $\rho \in$ $\mathcal{C}\left(\rho_{\mathrm{A}}\right)$. The definition (77) and the concavity of $S_{d}$ give $E^{c r}(\rho) \leq S_{d}\left(\rho_{\mathrm{A}}\right)$. If $d^{\prime} \geq d$, there are pure states $|\Psi\rangle$ of $\mathrm{AB}$ such that $\operatorname{tr}_{\mathrm{B}}|\Psi\rangle\langle\Psi|=\rho_{\mathrm{A}}$, and hence $E^{c r}(|\Psi\rangle\langle\Psi|)=$ $S_{d}\left(\rho_{\mathrm{A}}\right)$. Consequently, $\max _{\rho \in \mathcal{C}\left(\rho_{\mathrm{A}}\right)} E^{c r}(\rho)=S_{d}\left(\rho_{\mathrm{A}}\right)$.

We have thus, for a $d_{0}$-level system $\mathrm{A}$, the monogamy inequality

$$
E(\rho)+C_{d_{0}}\left(\rho_{\mathrm{A}}\right) \leq C_{d_{0}}^{\max },
$$

where $E$ is given by eq.(7) with the functions (6). Thus, the entanglement of $\mathrm{A}$ with $\mathrm{B}$, as quantified by $E(\rho)$, restricts the value of the left side of inequality (5). In particular, for a state $\rho$ such that $E(\rho) \geq C_{d_{0}}^{\max }-1$, this noncontextuality inequality cannot be violated. Equation (8) can also be read as an upper bound on the entanglement $E(\rho)$. In the extreme case of maximal violation of eq.(5), i.e., $C_{d_{0}}\left(\rho_{\mathrm{A}}\right)=C_{d_{0}}^{\max }$, it gives $E(\rho)=0$. There may be other entanglement monotones that coincide with the functions (6) when $\rho$ is pure, and so satisfy inequality (8). But, there is no entanglement monotone, for which eq.(8) is always an equality, since $C_{d_{0}}\left[\Lambda\left(\rho_{\mathrm{A}}\right)\right] \leq C_{d_{0}}\left(\rho_{\mathrm{A}}\right)$ for some local operations $\Lambda$ on A. Some noncontextuality inequalities (5) are violated for any state $\rho$, which, in this case, necessarily satisfies $E(\rho)<C_{d_{0}}^{\max }-1$. If the corresponding function $C_{d_{0}}$ is constant, $E=0$, and eq.(8) is trivially obeyed, and of no relevance. This is not surprising, since such a state-independent noncontextuality inequality is always maximally violated $[32-35]$. If $C_{d_{0}}$ is larger than unity, but not constant, eq.(8) still gives an upperbound, that depends on the entanglement beween $\mathrm{A}$ and $\mathrm{B}$, for the left side of eq.(5).

\section{COMPUTABLE MEASURES OF ENTANGLEMENT}

The monogamy inequality (8) involves an unusual entanglement monotone, defined from the considered noncontextuality inequality. Moreover, even familiar entanglement monotones are difficult to evaluate for an arbitrary density matrix $\rho$ [36]. An exception is the negativity $\left(\left\|\rho^{\Gamma}\right\|-1\right) / 2$, where $\|M\|=\operatorname{tr} \sqrt{M M^{\dagger}}$ denotes the trace norm of operator $M$, and $\rho^{\Gamma}$ is a partial transpose of $\rho[2,28,37]$. There are entangled states with vanishing negativity. Other quantities can be used to detect entanglement, e.g., $\|\mathcal{R}(\rho)\|$, where $\mathcal{R}$ is a matrix realignment map, which is not greater than 1 when $\rho$ is not entangled [38 -40]. In ref.[41], a lower bound is derived for the entanglement of formation, in terms of

$$
x \equiv \max \left\{\left\|\rho^{\Gamma}\right\|,\|\mathcal{R}(\rho)\|\right\},
$$

which is readily computable. We show below that a similar bound can be obtained for any entanglement monotone of the form (7).

Proposition 4. Consider an entanglement monotone $E^{c r}$ given by eq.(7), two systems, A and B, of Hilbert space dimensions $d$ and $d^{\prime}$, respectively, and the function $f$ defined, for $y \in\left[1, d^{*}\right]$, where $d^{*}=\min \left\{d, d^{\prime}\right\}$, by

$$
f(y) \equiv \inf _{\mathbf{p} \in \mathcal{F}(y)} s(\mathbf{p}),
$$

where $s$ is given by eq.(2), and $\mathcal{F}(y)$ is the set of the $d^{*}$-component probability vectors $\mathbf{p}$, such that $\left(\sum_{i=1}^{d^{*}} \sqrt{p_{i}}\right)^{2}=y$.

For any state $\rho$ of $\mathrm{AB}, E^{c r}(\rho) \geq c o(f)(x)$, where $c o(f)$ is the convex hull of $f$, and $x$ is given by eq.(9).

Proof. Let us first show that $c o(f)$ exists and is nondecreasing. Since $s$ is positive, $f \geq 0$, and thus, $f$ has a convex hull [42]. It is the maximum of the convex functions not larger than $f$. As $f \geq 0, c o(f)$ is positive. The only element of $\mathcal{F}(1)$ is $\mathbf{p}=1$. Thus, $f(1)=0$, and hence, $c o(f)(1)=0$. Consider $y_{1}$ and $y_{2}$ such that $1 \leq y_{1} \leq y_{2} \leq d^{*}$. We have $y_{1}=\tau+(1-\tau) y_{2}$ with $\tau \in[0,1]$. So, using the convexity and positivity of $c o(f)$, and $c o(f)(1)=0$, we get $c o(f)\left(y_{1}\right) \leq c o(f)\left(y_{2}\right)$.

Consider any ensemble $\left\{P_{m},\left|\Psi_{m}\right\rangle\right\} \in \mathcal{D}(\rho)$, and denote by $\mathbf{p}^{(m)}$ the $d^{*}$-component vector made up of the squared Schmidt coefficients of $\left|\Psi_{m}\right\rangle$, in decreasing order, possibly completed with zeros. By definition of $f, \sum_{m} P_{m} S_{d}\left(\operatorname{tr}_{\mathrm{B}} \rho_{m}\right) \geq \sum_{m} P_{m} f\left(y_{m}\right)$, where $\rho_{m}=$ $\left|\Psi_{m}\right\rangle\left\langle\Psi_{m}\right|$, and $y_{m}=\left[\sum_{i=1}^{d^{*}}\left(p_{i}^{(m)}\right)^{1 / 2}\right]^{2}$. The right side of this inequality is not smaller than $\operatorname{co}(f)(y)$ where $y=$ $\sum_{m} P_{m} y_{m}$. Since $y_{m}=\left\|\rho_{m}^{\Gamma}\right\|=\left\|\mathcal{R}\left(\rho_{m}\right)\right\|[28,38,43]$, and the trace norm is convex, $x \leq y$. Using this inequality, and the monotonicity of $c o(f)$, leads to the result.

The above proposition, and the monogamy inequality (8), give, for a $d_{0}$-level system $\mathrm{A}$,

$$
C_{d_{0}}\left(\rho_{\mathrm{A}}\right) \leq C_{d_{0}}^{\max }-\operatorname{co}(f)(x),
$$

where $f$ is given by eq.(10) with the functions (6), and $d^{*}=\min \left\{d_{0}, d^{\prime}\right\}$ with $d^{\prime}$ the Hilbert space dimension of B. For states $\rho$ such that $c o(f)(x) \geq C_{d_{0}}^{\max }-1$, eq.(5) cannot be violated. As noted above, if all products in eq.(5), have an even number of terms, $C_{d} \geq 1$, and so, $c o(f)$ can reach $C_{d_{0}}^{\max }-1$ only for $x=d^{*}$, i.e., for maximally entangled states. However, even in this case, eq.(11) can be useful to determine states too entangled to violate inequality (5). As an example, consider $d^{*}=d_{0}=4$, and the CHSH inequality, for which, as shown below, $C_{4}\left(\rho_{\mathrm{A}}\right)=\max \left\{1, C_{4}^{\prime}\left(\rho_{\mathrm{A}}\right)\right\}$, where

$$
C_{4}^{\prime}\left(\rho_{\mathrm{A}}\right)=\sqrt{2\left[\left(p_{1}-p_{4}\right)^{2}+\left(p_{2}-p_{3}\right)^{2}\right]},
$$


with $p_{i}=\lambda_{i}\left(\rho_{\mathrm{A}}\right)$. The functions (6), defined with $C_{4}^{\prime}$, have all the necessary properties to obey eq.(11) with the corresponding $f$. Using the method of Lagrange multipliers, we find

$f(y)=\sqrt{2}-(3 \sqrt{y}+\sqrt{32-7 y})^{3 / 2}(\sqrt{32-7 y}-\sqrt{y})^{1 / 2} / 32$.

This function is convex, and is hence equal to its convex hull. It increases from 0 to $C_{4}^{\max }=\sqrt{2}$. Consequently, for states $\rho$ such that $x \geq 2.95, C_{4}^{\prime}\left(\rho_{\mathrm{A}}\right) \leq 1$, and thus, the local CHSH inequality is always satisfied.

Proof. For CHSH inequality, $C_{d}\left(\rho_{\mathrm{A}}\right)=\sup _{\mathbf{A} \in \mathcal{A}_{d}}\langle T\rangle / 2$, where $\langle T\rangle=\operatorname{tr}\left(\rho_{\mathrm{A}} T\right)$, and $T=A_{1}\left(A_{2}+A_{4}\right)+A_{3}\left(A_{2}-\right.$ $\left.A_{4}\right)$. For $A_{k}=A_{1},\langle T\rangle=2$, and hence $C_{d} \geq 1$. We are thus interested only in observables $A_{k}$ such that $\langle T\rangle$ can be larger than 2. For $d=4, A_{k}$ can be written as $A_{k}=\eta_{k}\left(2 \Pi_{k}-I\right)$ where $\eta_{k}= \pm 1, I$ is the identity operator, and $\Pi_{k}$ is a projector of rank not greater than 2 . Using this expression, one finds $T^{2}=4 I \pm 16 R$, where $R=\left[\Pi_{1}, \Pi_{3}\right]\left[\Pi_{2}, \Pi_{4}\right]$ [44]. If $R=0$, the eigenvalues of $T$ can only be 2 and -2 , and so $\langle T\rangle \leq 2$. We thus search for the projectors $\Pi_{k}$ for which $R \neq 0$. If two commuting $\Pi_{k}$ and $\Pi_{l}$, obey $\Pi_{k} \Pi_{l}=0$ or $\Pi_{k} \Pi_{l}=\Pi_{k}$, then $R=0$. Consequently, the sought projectors are of rank 2 , and such that, for $\left[\Pi_{k}, \Pi_{l}\right]=0, \Pi_{k} \Pi_{l}$ is a rank-1 projector. This gives $\Pi_{k}=|k\rangle\left\langle k|+| k^{\prime}\right\rangle\left\langle k^{\prime}\right|$, with

$$
\begin{gathered}
|1\rangle=|\tilde{1}\rangle,\left|1^{\prime}\right\rangle=|2\rangle=|\tilde{2}\rangle,\left|2^{\prime}\right\rangle=|\tilde{3}\rangle,|3\rangle=\nu_{1}|\tilde{2}\rangle+\hat{\nu}_{1}|\tilde{3}\rangle, \\
\left|3^{\prime}\right\rangle=\nu_{1}|\tilde{1}\rangle+\hat{\nu}_{1}|\tilde{4}\rangle,|4\rangle=\nu_{2}|\tilde{1}\rangle+\hat{\nu}_{2}|\tilde{2}\rangle,\left|4^{\prime}\right\rangle=\hat{\nu}_{2}|\tilde{3}\rangle+\nu_{2}|\tilde{4}\rangle,
\end{gathered}
$$

where $\{|\tilde{\imath}\rangle\}_{i=1}^{4}$ is any orthonormal basis, $\left|\hat{\nu}_{k}\right|^{2}+\nu_{k}^{2}=1$, and $\nu_{k} \in[0,1]$. For these projectors, the eigenvalues of $\left[\Pi_{k}, \Pi_{k+2}\right]$, where $k=1$ or 2 , are $\pm i \nu_{k}\left|\hat{\nu}_{k}\right|$. Since these two commutators commute with each other, and $\operatorname{tr} R=0$, there are $A_{k}$ such that $\boldsymbol{\lambda}(R)=4 r(1,1,-1,-1)$, where $r \in] 0,1]$. For these $A_{k}, \operatorname{tr}\left(A_{k} A_{l}\right)=0$ for commuting $A_{k}$ and $A_{l}$, and hence $\operatorname{tr} T=0$. So, $\boldsymbol{\lambda}(T)=$ $\left(r_{+}, r_{-},-r_{-},-r_{+}\right)$, where $r_{ \pm}=2 \sqrt{1 \pm r}$. Maximising $\boldsymbol{\lambda}(T) \cdot \boldsymbol{p}$ over $r$, leads to eq.(12).

\section{CONCLUSION}

In summary, a monogamy inequality for entanglement and local contextuality, has been derived. It involves an entanglement monotone that depends on the considered noncontextuality inequality, and the Hilbert space dimension of the local system. It essentially results from the relations between the entanglement of the global state and the entropy of the local state, and between the eigenvalues of the local state and its ability to disobey the noncontextuality inequality. Thus, other entanglement monotones, different from the one we have built, may satisfy the same monogamy inequality. A consequence of the found monogamy, is that there are global states so entangled that they cannot violate the noncontextuality inequality. The obtained monogamy inequality relates entanglement per se to local contextuality. It would thus be of interest to find out if there are global states, that are Bell-local [11], but still entangled enough to prevent the violation of a local noncontextuality inequality.
[1] V. Coffman, J. Kundu, and W.K. Wootters, Distributed entanglement, Phys. Rev. A 61, 052306 (2000).

[2] R. Horodecki, P. Horodecki, M. Horodecki, and K. Horodecki, Quantum entanglement, Rev. Mod. Phys. 81, 865 (2009).

[3] G. Vidal, Entanglement monotones, J. Mod. Opt. 47, 355 (2000).

[4] M. B. Plenio, and S. Virmani, An introduction to entanglement measures, Quantum Inf. Comput. 7, 1 (2007).

[5] M. Koashi, and A. Winter, Monogamy of quantum entanglement and other correlations, Phys. Rev. A 69, 022309 (2004).

[6] T.J. Osborne, and F. Verstraete, General monogamy inequality for bipartite qubit entanglement, Phys. Rev. Lett. 96, 220503 (2006).

[7] Y.-K. Bai, Y.-F. Xu, and Z.D. Wang, General Monogamy Relation for the Entanglement of Formation in Multiqubit Systems, Phys. Rev. Lett. 113, 100503 (2014).

[8] W. Song, Y.-K. Bai, M. Yang, M. Yang, and Z.-L. Cao, General monogamy relation of multi-qubit systems in terms of squared Rényi-alpha entanglement, Phys. Rev. A 93, 022306 (2016).

[9] Y. Luo, T. Tian, L.-H. Shao, and Y. Li, General
Monogamy of Tsallis-q Entropy Entanglement in Multiqubit Systems, Phys. Rev. A 93, 062340 (2016).

[10] C. Lancien, S. Di Martino, M. Huber, M. Piani, G. Adesso, and A. Winter, Should Entanglement Measures be Monogamous or Faithful ?, Phys. Rev. Lett. 117, 060501 (2016).

[11] J.S. Bell, On the Einstein Podolsky Rosen paradox, Physics (Long Island City, N.Y.) 1, 195 (1964).

[12] J.F. Clauser, M.A. Horne, A. Shimony, and R.A. Holt, Proposed experiment to test local hidden-variable theories, Phys. Rev. Lett. 23, 880 (1969).

[13] B. Toner, Monogamy of non-local quantum correlations, Proc. R. Soc. A 465, 59 (2009).

[14] B. Toner, and F. Verstraete, Monogamy of Bell correlations and Tsirelson's bound, arXiv:quant-ph/0611001.

[15] A.A. Klyachko, M.A. Can, S. Binicioğlu, and A.S. Shumovsky, Simple Test for Hidden Variables in Spin-1 Systems, Phys. Rev. Lett. 101, 020403 (2008).

[16] P. Kurzyński, A. Cabello, and D. Kaszlikowski, Fundamental Monogamy Relation between Contextuality and Nonlocality, Phys. Rev. Lett. 112, 100401 (2014).

[17] D. Saha, and R. Ramanathan, A unified graph-theoretic approach to monogamy relations between nonlocality and 
contextuality, arXiv:1606.04021

[18] P. Kurzyński and D. Kaszlikowski, Contextuality of almost all qutrit states can be revealed with nine observables, Phys. Rev. A 86, 042125 (2012).

[19] Z.-P. Xu, H.-Y. Su, and J.-L. Chen, Quantum contextuality of a qutrit state, Phys. Rev. A 92, 012104 (2015).

[20] S. Camalet, Simple state preparation for contextuality tests with few observables, Phys. Rev. A 94, 022106 (2016).

[21] R. Ramanathan and P. Horodecki, Necessary and Sucient Condition for State-Independent Contextual Measurement Scenarios, Phys. Rev. Lett. 112, 040404 (2014).

[22] G.H. Hardy, J.E. Littlewood, and G. Pólya, Inequalities (Cambridge University Press, Cambridge, 1952).

[23] A. W. Marshall, I. Olkin, and B.C. Arnold, Inequalities: Theory of Majorization and its Applications, Second edition, Springer Series in Statistics (Springer, New York, 2011).

[24] G.M. Bosyk , S. Zozor, F. Holik, M. Portesi and P.W. Lamberti, A family of generalized quantum entropies: definition and properties, Quant. Inf. Proc. 15, 3393 (2016).

[25] C.H. Bennett, H. J. Bernstein, S. Popescu, and B. Schumacher, Concentrating partial entanglement by local operations, Phys. Rev. A 53, 2046 (1996).

[26] V. Vedral, and M. B. Plenio, Entanglement measures and purification procedures, Phys. Rev. A 57, 1619 (1998).

[27] G. Vidal, and R. Tarrach, Robustness of entanglement, Phys. Rev. A 59, 141 (1999).

[28] G. Vidal and R.F. Werner, Computable measure of entanglement, Phys. Rev. A 65, 032314 (2002).

[29] M.A. Nielsen, Conditions for a Class of Entanglement Transformations, Phys. Rev. Lett. 83, 436 (1999).

[30] A.I. Khinchin, Mathematical Foundations of Information Theory (Dover, New York, 1957).

[31] M. Araújo, M.T. Quintino, C. Budroni, M.T. Cunha, and A. Cabello, All noncontextuality inequalities for the n-cycle scenario, Phys. Rev. A 88, 022118 (2013).

[32] A. Peres, Incompatible results of quantum measurements, Phys. Lett. A 151, 107 (1990).

[33] N. D. Mermin, Simple Unified Form for the Major NoHidden-Variables Theorems, Phys. Rev. Lett. 65, 3373 (1990).

[34] A. Cabello, Experimentally Testable State-Independent Quantum Contextuality, Phys. Rev. Lett. 101, 210401 (2008).

[35] S. Yu and C. Oh, State-Independent Proof of Kochen Specker Theorem with 13 Rays, Phys. Rev. Lett. 108, 030402 (2012).

[36] Y. Huang, Computing quantum discord is NP-complete, New J. Phys. 16, 033027 (2014).

[37] K. Życzkowski, P. Horodecki, A. Sanpera and M. Lewenstein, Volume of the set of separable states, Phys. Rev. A 58, 883 (1998).

[38] O. Rudolph, Further results on the cross norm criterion for separability, arXiv:quant-ph/0202121

[39] O. Rudoplh, Some properties of the computable crossnorm criterion for separability, Phys. Rev. A 67, 032312 (2003).

[40] K. Chen, and L.-A. Wu, A matrix realignment method for recognizing entanglement, Quantum Inf. Comput. 3, 193 (2003).

[41] K. Chen, S. Albeverio, and S.-M. Fei, Entanglement of Formation of Bipartite Quantum States, Phys. Rev. Lett. 95, 210501 (2005).

[42] J.-B. Hiriart-Urruty, and C. Lemaréchal, Fundamentals of Convex Analysis (Springer, Berlin, 2001).

[43] K. Chen, S. Albeverio, and S.-M. Fei, Concurrence of Arbitrary Dimensional Bipartite Quantum States, Phys. Rev. Lett. 95, 040504 (2005).

[44] L.J. Landau, On the violation of Bell inequality in quantum theory, Phys. Lett. A 120, 54 (1987). 